\title{
Wake measurements of a dechirper jaw with nonzero tilt angle
}

\author{
Karl Bane, Marc Guetg, and Alberto Lutman \\ SLAC National Accelerator Laboratory, Menlo Park, California 94025, USA
}

(Received 8 January 2018; published 29 May 2018)

\begin{abstract}
The RadiaBeam/SLAC dechirper at the Linac Coherent Light Source (LCLS) is being used as a fast kicker, by inducing transverse wakefields, to, e.g., facilitate Fresh-slice, two-color laser operation. The dechirper jaws are independently adjustable at both ends, and it is difficult to avoid leaving residual (longitudinal) tilt in them during setup. In this report we develop a model independent method of removing unknown tilt in a jaw. In addition, for a short uniform bunch passing by a single dechirper plate, we derive an explicit analytical formula for the transverse wake kick as function of average plate offset and tilt angle. We perform wake kick measurements for the different dechirper jaws of the RadiaBeam/SLAC dechirper, and find that the agreement between measurement and theory is excellent.
\end{abstract}

DOI: 10.1103/PhysRevAccelBeams.21.054402

\section{INTRODUCTION}

The Linac Coherent Light Source (LCLS) at SLAC is a linac-based, free electron laser (FEL), that generates X-rays with wavelengths down to 1 angstrom [1]. The RadiaBeam/ SLAC dechirper at the LCLS is a corrugated structure that is being used as a passive fast kicker, to facilitate the Freshslice, two-color scheme of generating x-rays [2]. In this mode of operation, after the final linac, the beam is made to pass near one jaw of a dechirper module, in order to send the tail of the bunch on a different trajectory than the head on the way to the undulator. During alignment of the jaws, each end is moved independently by two motors. At the end, in general, a jaw will tend to have an offset as well as some residual tilt with respect to the beam trajectory.

Typically, for fresh-slice, two-color operation the dechirper jaw is moved toward the beam and adjusted while observing the size of the effect on the induced, downstream oscillation of the beam. The adjustment is not precise and is done somewhat by feel. There may come a time, however, when it is important to accurately know the location and orientation of the jaw with respect to the beam. In a recent report on wakefield measurements on the dechirper at the LCLS, the agreement between measurement and calculation was found to be excellent, after a slight adjustment to the gap parameter in the theory (in twoplate measurements) $[3,4]$. However, because of the possibility of an unknown tilt in the jaws, one could not simply

\footnotetext{
*kbane@slac.stanford.edu

Published by the American Physical Society under the terms of the Creative Commons Attribution 4.0 International license. Further distribution of this work must maintain attribution to the author(s) and the published article's title, journal citation, and DOI.
}

conclude that the discrepancy implied an error in measurement or theory.

This report uses a model independent method of removing unknown tilt in a jaw of the RadiaBeam/SLAC dechirper. The idea of the method is simple. The average transverse kick (or center of mass kick) experienced by a beam on passing by a dechirper plate depends on a strong inverse power of the offset of beam from plate (minus the third power for short bunches). If we run a procedure that fixes the beam offset at the center of the plate (longitudinally, in $z$ ) while varying the tilt angle in both positive and negative directions, the average wake kick will trace out a curve that has a minimum at the condition of zero tilt angle. This is precisely the experiment that we have performed and report on here.

In this report we also develop an analytical formula for the wake kick experienced by a short bunch on passing a single dechirper plate, as function of average beam offset and plate tilt angle. This allows us to perform a more precise comparison with measurement than was done before, where we assumed that the tilt angle was small and could be ignored $[3,4]$. Note that other reports on wake measurements of such corrugated structures are found in the literature [5-8], though where bunches of much lower energies and longer lengths were used.

This report is organized as follows: we begin with Sec. II, the theory section. where we derive the average transverse kick experienced by a short, ultrarelativistic bunch passing by a single dechirper plate, as function of average beam offset and plate tilt. Section III gives a discussion of two approaches to the measurements. Section IV, the measurement section proper, describes how the measurements were performed, presents the data, and compares to theory. Section V gives the conclusions.

The measurements presented here originally contained both longitudinal and transverse kick data; the longitudinal 
data, however, was of poor quality and will not be shown. Nevertheless, in Appendix A we derive the average longitudinal kick experienced by a short bunch passing by a single dechirper plate, as function of average beam offset and plate tilt. In Appendix B we offer more details of the measurement layout and the measurements.

\section{THEORY}

The geometry of three corrugations of a horizontal $(x)$ dechirper module of the RadiaBeam/SLAC dechirper is shown in Fig. 1, with the parameters given in Table I. We present here the theory for a beam passing by a single jaw of an $x$ dechirper module; the theory for a $y$ dechirper module is exactly the same, since the only difference in geometry is a $90^{\circ}$ rotation about the beam axis.

Let us consider the case of a beam passing by a single dechirper jaw, with the other jaw far away and not interacting with the beam. The ends of the dechirper jaws are independently adjustable. Thus, in general, the configuration of beam and jaw can be characterized by two parameters, average offset $b$ and extra offset at the jaw ends $\pm d$ (see Fig. 2; or, equivalently, jaw tilt angle $\tan \theta=2 d / L \approx \theta$, with $L$ the dechirper jaw length).

In the measurements to be presented below, the wake strength is quantified by the average transverse kick induced in the beam during its passage near a jaw; this quantity is proportional to the average of the bunch wake, i.e., the kick factor, $\varkappa_{x}$. The bunch at the end of the LCLS linacs is short with an approximately uniform distribution. The kick factor for a short, uniform bunch of full length $\ell$, passing by a single dechirper plate at offset $b$ (with no tilt; here short means $\ell \ll b)$, is $[4,9,10]$

$$
\varkappa_{x}(b)=\left(\frac{Z_{0} c}{4 \pi b^{3}}\right) s_{0 x} f_{x}\left(\frac{\ell}{s_{0 x}}\right)
$$

with $Z_{0}=377 \Omega, c$ the speed of light; where

$$
s_{0 x}=\frac{8 b^{2} t}{9 \pi \alpha^{2} p^{2}},
$$

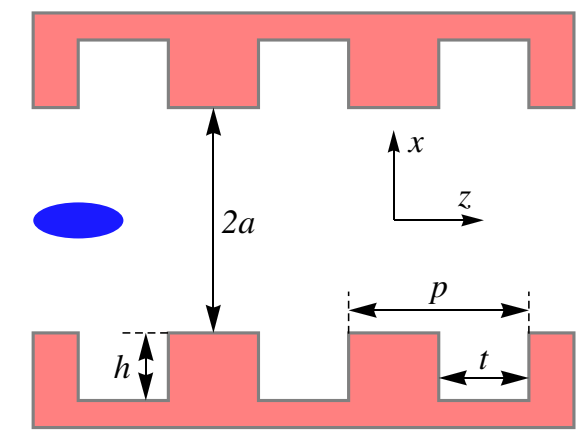

FIG. 1. Geometry of a (horizontal) dechirper module showing three corrugations. The blue ellipse represents an electron beam propagating along the $z$ axis.
TABLE I. Parameters of the RadiaBeam/SLAC dechirper.

\begin{tabular}{lcc}
\hline \hline Parameter & Value & Unit \\
\hline Half gap $a$ & $0.5-12.5$ & $\mathrm{~mm}$ \\
Corrugation properties: & & \\
Period $p$ & 0.5 & $\mathrm{~mm}$ \\
Depth $h$ & 0.5 & $\mathrm{~mm}$ \\
Longitudinal gap $t$ & 0.25 & $\mathrm{~mm}$ \\
Plate width $w$ & 12 & $\mathrm{~mm}$ \\
Length of structure $L$ & 2 & $\mathrm{~m}$ \\
\hline \hline
\end{tabular}

with $\alpha=1-0.465 \sqrt{t / p}-0.070(t / p)$, and

$f_{x}(\zeta)=1-\frac{12}{\zeta}+\frac{120}{\zeta^{2}}-8 e^{-\sqrt{\zeta}}\left(\frac{1}{\zeta^{1 / 2}}+\frac{6}{\zeta}+\frac{15}{\zeta^{3 / 2}}+\frac{15}{\zeta^{2}}\right)$.

The dechirper plate tilt angle is necessarily small, on the order of 1 milliradian or less. For a plate with a small-angle tilt, with average offset $b$ and offset at the two ends of $b-d$ and $b+d$, we approximate the total kick factor by adiabatically averaging Eq. (1) along the beam's path past the plate:

$$
\varkappa_{x t}(b, d)=\frac{1}{2 d} \int_{b-d}^{b+d} \varkappa_{x}\left(b^{\prime}\right) d b^{\prime} .
$$

Substituting in Eq. (1), and performing the integral we obtain

$$
\varkappa_{x t}(b, d)=\left(\frac{Z_{0} c}{8 \pi d}\right)[g(b+d)-g(b-d)],
$$

with

$$
\begin{aligned}
g(x)= & \frac{8 t}{9 \pi \alpha^{2} p^{2} \xi^{2}}\left[6 x^{2}\left(5 x^{2}-\xi\right)+e^{-\xi^{1 / 2} / x}\left(x \xi^{3 / 2}-9 x^{2} \xi\right.\right. \\
& \left.\left.-30 x^{3} \xi^{1 / 2}-30 x^{4}\right)+\xi^{2}\left(E i\left[-\xi^{1 / 2} / x\right]+\ln x\right)\right] ;
\end{aligned}
$$

where

$$
\xi=\frac{9 \pi \alpha^{2} p^{2} \ell}{8 t}
$$

and $\operatorname{Ei}(x)$ is the exponential integral function. Note that $\varkappa_{x t}(b, d)$. as required, is symmetric with respect to the

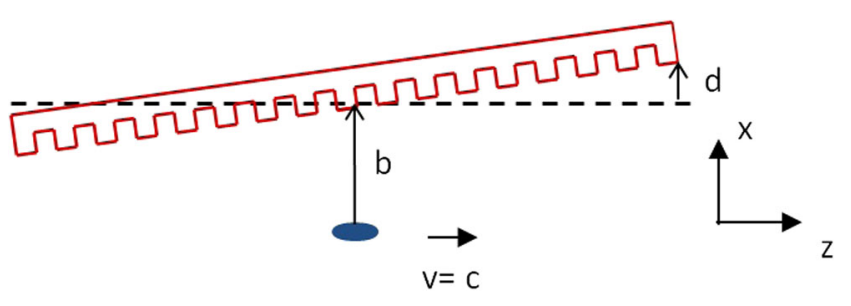

FIG. 2. Sketch of orientation of beam and jaw during measurement (for a horizontal jaw). The beam (blue ellipse) moves in the $z$ direction below the dechirper jaw (red), at average offset $b$; the jaw tilt (with respect to $z$ ) is defined by the change in offsets at the jaw ends, $\pm d$. Note that the corrugation size and tilt angle as sketched are much larger than they are in reality. 


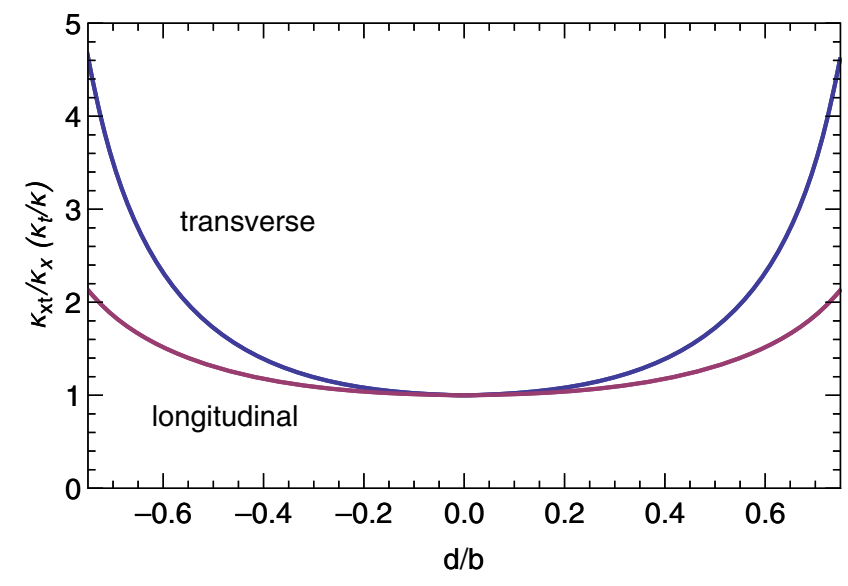

FIG. 3. The kick factor for the tilted-plate configuration normalized to the non-tilted case, $\varkappa_{x t}(b, d) / \varkappa_{x}(b)$, as function of $d / b$ (blue). Here we have used as corrugation parameters those of the RadiaBeam/SLAC dechirper, full bunch length $\ell=18 \mu \mathrm{m}$, and average offset of plate from beam, $b=1 \mathrm{~mm}$. For the longitudinal case, the change in relative loss factor, $\varkappa_{t}(b, d) / \varkappa(b)$, derived in Appendix $\mathrm{A}$, is given in red.

variable $d$. In Appendix A we derive the corresponding equation for the average longitudinal wake of a tilted plate.

The kick factor for the tilted configuration normalized to the nontilted case, $\varkappa_{x t}(b, d) / \varkappa_{x}(b)$, as function of $d / b$ is shown in Fig. 3 (in blue). Here we have used as corrugation parameters those of the RadiaBeam/SLAC dechirper, as bunch distribution one that is uniform with full length $\ell=18 \mu \mathrm{m}$, and as average offset of plate from beam, $b=1 \mathrm{~mm}$. For comparison, we present also the longitudinal effect, i.e., the change in relative loss factor, $\varkappa_{t} / \varkappa_{\text {, }}$ which is given in red. We see that the longitudinal wake is a less sensitive function of the tilt than the transverse wake. This is because the longitudinal wake has a weaker dependence on offset of beam from plate, $b$; for a short bunch it varies as $b^{-2}$ instead of the $b^{-3}$ of the transverse case [10].

The beam position at downstream beam position monitor (BPM) - assuming the beam is initially traveling parallel to the $z$-axis and that there is no intervening magnet-is simply

$$
x_{b}=e Q L L_{\mathrm{BPM} \varkappa_{x t}}(b, d) / E
$$

with $Q$ beam charge, $L$ length of dechirper plate, $L_{\mathrm{BPM}}$ distance between dechirper and measuring BPM, and $E$ beam energy (and the equivalent formula holds for the offset after a $y$ dechirper jaw, $y_{b}$ ). Of course, a usual implicit assumption is that the tail of the bunch does not move significantly, compared to offset $b$, during the traversal past the plate.

\section{APPROACH TO MEASUREMENT}

In Ref. [4] single plate wake measurements of the SLAC/ RadiaBeam dechirper were performed and compared with theory. There the plate was assumed to have negligible tilt and the transverse wake kick was measured as function of plate offset from the beam, $b$. Simultaneously, the longitudinal wake effect was also measured as function of $b$. As will also be done here, it was assumed that the measured offsets $b$ contained an unknown overall shift $b_{0}$ that could be significantly larger than the relative error between two $b$ settings. The fact that theory and measurement agreed well, and that the fitted $b_{0}$ agreed well for both jaw measurements, gave the authors confidence that the jaw tilt for these measurements was indeed small, and that the measurements confirmed the theory.

In the present report we only consider transverse measurements, and we compare them with the analytical function that includes both the effects of offset $b$ and tilt parameter $d$, Eqs. (5) and (8). We do not vary $b$ during the measurement, but rather keep $b$ fixed and vary $d$ (as is suggested by the blue curve in Fig. 3). This has a clear advantage: A single plate has no symmetry axis (in offset), and measuring kick while varying $b$ will yield nonsymmetric results. However, a measurement of kick where we only vary $d$, in theory, gives a symmetric result. Thus, simply from seeing a plot of the data, one can gauge its quality (by how symmetric it is); in addition, one knows that the location of the minimum of the curve corresponds to the condition of the plate having zero tilt.

A second advantage of this measurement approach has to do with correlations. Consider Fig. 4, which simulates the earlier type of kick measurement, where the beam offset $b$ is varied without changing the tilt. (The corrugation parameters used were those of the RadiaBeam/SLAC dechirper; the bunch distribution assumed was uniform of full length $\ell=18 \mu \mathrm{m}$.) The blue curve gives the kick factor assuming no tilt in the jaw. On the same plot we present results for tilt parameter $d=0.2 \mathrm{~mm}$ (red dashes),

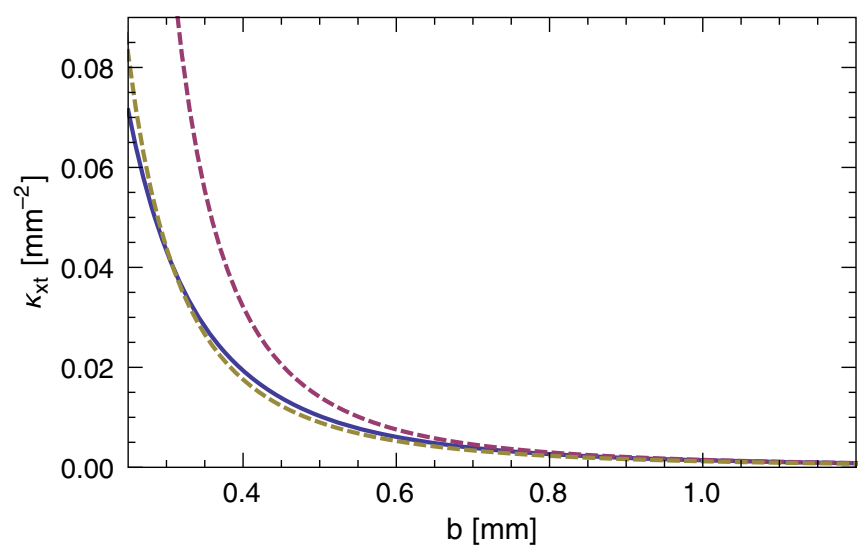

FIG. 4. The calculated kick factor $\varkappa_{x t}(b, d)$ as function of beam offset $b$ assuming no tilt in the jaw (blue solid curve). For comparison, the results for tilt parameter $d=0.2 \mathrm{~mm}$ are given in red dashes; those for tilt parameter $d=0.2 \mathrm{~mm}$ and shift by offset parameter $b_{0}=-70 \mu \mathrm{m}$ are given in gold dashes. The corrugation parameters used were those of the RadiaBeam/SLAC dechirper; the bunch distribution assumed was uniform of full length $\ell=18 \mu \mathrm{m}$. 


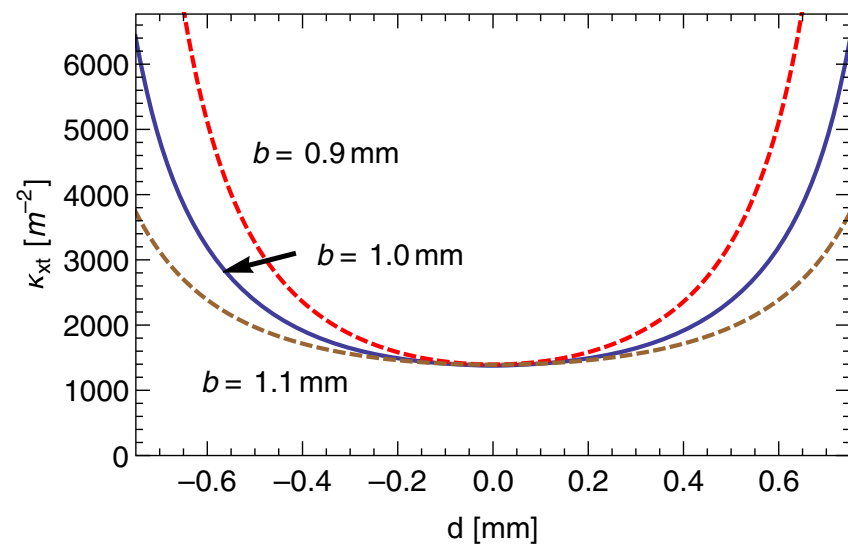

FIG. 5. The calculated kick factor $\varkappa_{x t}(b, d)$ as function of tilt parameter $d$ for offset parameter $b=1.0 \mathrm{~mm}$ (blue solid curve). For comparison, the results for $b=0.9 \mathrm{~mm}$ (red dashes) and $b=1.1 \mathrm{~mm}$ (brown dashes) are also shown. To emphasize the difference in curvature of the three curves, the latter two were shifted vertically (by, respectively, $-480 \mathrm{~m}^{-2},+350 \mathrm{~m}^{-2}$ ) to give the same minimum. The corrugation parameters used were those of the RadiaBeam/SLAC dechirper; the bunch distribution assumed was uniform of full length $\ell=18 \mu \mathrm{m}$.

which differ significantly from the blue curve. However, when we shift the abscissa values of these last results by $b_{0}=-70 \mu \mathrm{m}$, we obtain the curve of the gold dashes, which is now close to the blue curve. Thus, there is significant correlation between the parameters $d$ and $b_{0}$, and using them as fitting parameters for such a measurement will not reliably find their separate values. Consequently, this kind of measurement is not a good way to find the offset and tilt of a dechirper plate.

In contrast, consider Fig. 5, which is a simulation of the type of measurement described in the present report, where only tilt parameter $d$ is varied. We plot $\varkappa_{x t}$ vs $d$ for cases average offset $b=0.9 \mathrm{~mm}$ (red dashes), $1.0 \mathrm{~mm}$ (blue solid line), and $1.1 \mathrm{~mm}$ (brown dashes). The dashed curves have been shifted vertically so that all have the same minimum value. One can see that the curvature of the curves varies inversely with average offset $b$, and that variations in $b$ and $d$ can be easily distinguished.

\section{MEASUREMENTS}

\section{A. Description}

The RadiaBeam/LCLS dechirper installed in the LCLS consists of a vertical module followed by a horizontal one. The jaws are denoted as "Top" and "Bottom" for the vertical module, and "North" and "South" for the horizontal one. The measurement was performed using a computer script that stepped through a symmetric range of tilt parameter $d$ keeping the average offset $b$ fixed. Figure 9 in Appendix B gives a sketch of the adjustment system for each dechirper jaw, which comprises two movers ("M" in the figure). The main mover (at top center) shifts the entire jaw without changing its inclination (or tilt) angle; the trim mover (the other mover in the figure) shifts the downstream end of the jaw. Adjustment of the tilt angle while keeping the average distance to the beam constant, therefore, requires the actuation of both motors. Due to mechanical backlash this can lead to discrepancies between set-points (requested settings) and actual position values. For our measurements, this effect was mitigated by actuating the motors always in one direction.

For the measurements described below, the beam was kept steady and the dechirper jaws were moved. For each data set one jaw was moved toward the beam trajectory, following the sequence the horizontal jaws-North then South-followed by the vertical jaws-Top then Bottom. During each measurement the tilt parameter, $d$, was changed while trying to keep the average offset of the jaw, $b$, fixed. The wake effect was measured using a downstream beam position monitor, BPM 590.

During the measurements the charge $Q=160 \mathrm{pC}$, beam energy $E=13.24 \mathrm{GeV}$, and distance between dechirper and measuring $\mathrm{BPM}, L_{\mathrm{BPM}}=16.26 \mathrm{~m}$. In Fig. 6 we display the bunch distribution, with the head to the left, as obtained by the transverse cavity, XTCAV [11]. We see that the distribution is approximately uniform; the uniform distribution with the same area and rms length has peak current $I=2.7 \mathrm{kA}$ and full length $\ell=18 \mu \mathrm{m}$. The transverse beam sizes at the dechirper are $\sigma_{x}=14 \mu \mathrm{m}$, $\sigma_{y}=40 \mu \mathrm{m}$; our theory assumes that the beam size is small compared to the distance between beam and plate, which is satisfied for our measurements.

During data taking, at each stop of the movers, about 100-200 measurements of the BPM 590 reading, $x_{b}$ (for North and South) or $y_{b}$ (for Top and Bottom), were obtained. For the measurements at each plate, the theory [Eqs. (5) and (8)] was fit to the data using a nonlinear model fit with three fitting parameters: average offset of beam from jaw $b$, shift in tilt parameter $d_{0}$, and shift in downstream offset of beam, $x_{b 0}$ or $y_{b 0}$. The function variable in the fit was tilt parameter $d$.

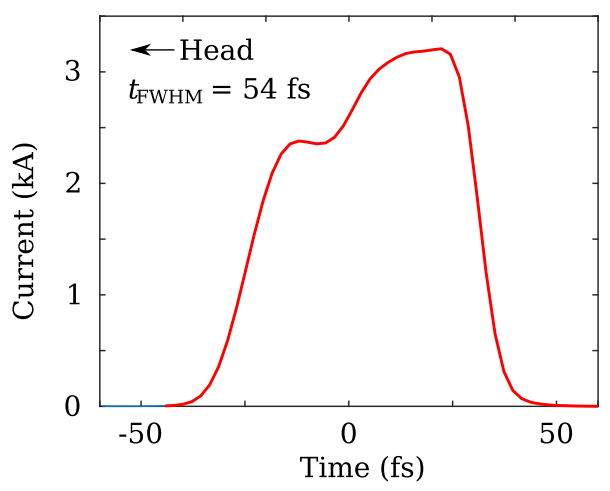

FIG. 6. The bunch distribution as obtained by the transverse cavity, XTCAV. The head of the bunch is to the left. 
Each dechirper plate has two LVDT (linear variable differential transformer) position sensors located at the plate ends (see Fig. 9 in Appendix B). These give us a second, more accurate way to compare the measurements with theory, in the event the movers-because of e.g., backlash in the motors-do not manage to keep $b$ constant during a data taking sequence. For the $i$ th measurement stop at any plate, we can convert the two LVDT readings to tilt parameter $d_{i}$ and average offset parameter $\delta b_{i}$, where the total average offset between beam and plate at stop $i$ becomes

$$
b_{i}=b_{0}+\delta b_{i} .
$$

The data now becomes the pair of coordinates $\delta b_{i}, d_{i}$. To fit to the data we again use a nonlinear model fit with three fitting parameters: shift in average offset of beam from jaw $b_{0}$, shift in tilt parameter $d_{0}$, and shift in downstream offset of beam, $x_{b 0}$ or $y_{b 0}$. The fit function now has two variables $\delta b$ and $d$. Note that, if the movers positioned the dechirper plate precisely as desired, then the above two fitting methods should give the same result. For the data to be presented below, we will see that the two methods agree quite well.

\section{B. Results}

The North results are given in Fig. 7 (the top plot), showing the beam offset at the measuring BPM, $x_{b}$ vs tilt parameter, $d$. The blue plotting symbols in this and following figures give the measurement points, after they have been shifted vertically by $-x_{b 0}$, horizontally by $-d_{0}$ (discussed more below). For each abscissa value, the rms deviation in measured $x_{b}$ is $\sigma \sim 10 \mu \mathrm{m}$, and an estimate of the measurement error, $\sigma / \sqrt{N_{m}} \sim 1 \mu \mathrm{m}$, where $N_{m}$ is the number of measurements. In this and following figures the data is given with error bars, showing the estimate of measurement error (which however here is tiny and not visible). The data looks good in that it traces out a curve that is very symmetric about a minimum.

The single variable fit to the data gives the red curve in the plot, with fitting parameters: average offset $b=0.70 \mathrm{~mm}$, tilt parameter shift $d_{0}=-0.42 \mathrm{~mm}$, and shift in BPM measurement $x_{b 0}=-8 \mu \mathrm{m}$. For the more accurate, two variable fit, first note that Fig. 10 in Appendix B shows the $\delta b_{i}$ and $d_{i}$ obtained from the LVDT readings for all the data taken. We see that there is little variation in the $\delta b_{i}$ in the North data (the blue plotting symbols). The variation that we do see is likely caused by mechanical backlash in the movers.

The results of the two variable fit for the North jaw are given by the red plotting symbols in Fig. 7 (the top plot). (Note that for every blue symbol there is a red symbol; some, however, are obscured by the corresponding blue symbol and are difficult to see.) A red symbol that does not fall on the symmetric red curve- such as the one at
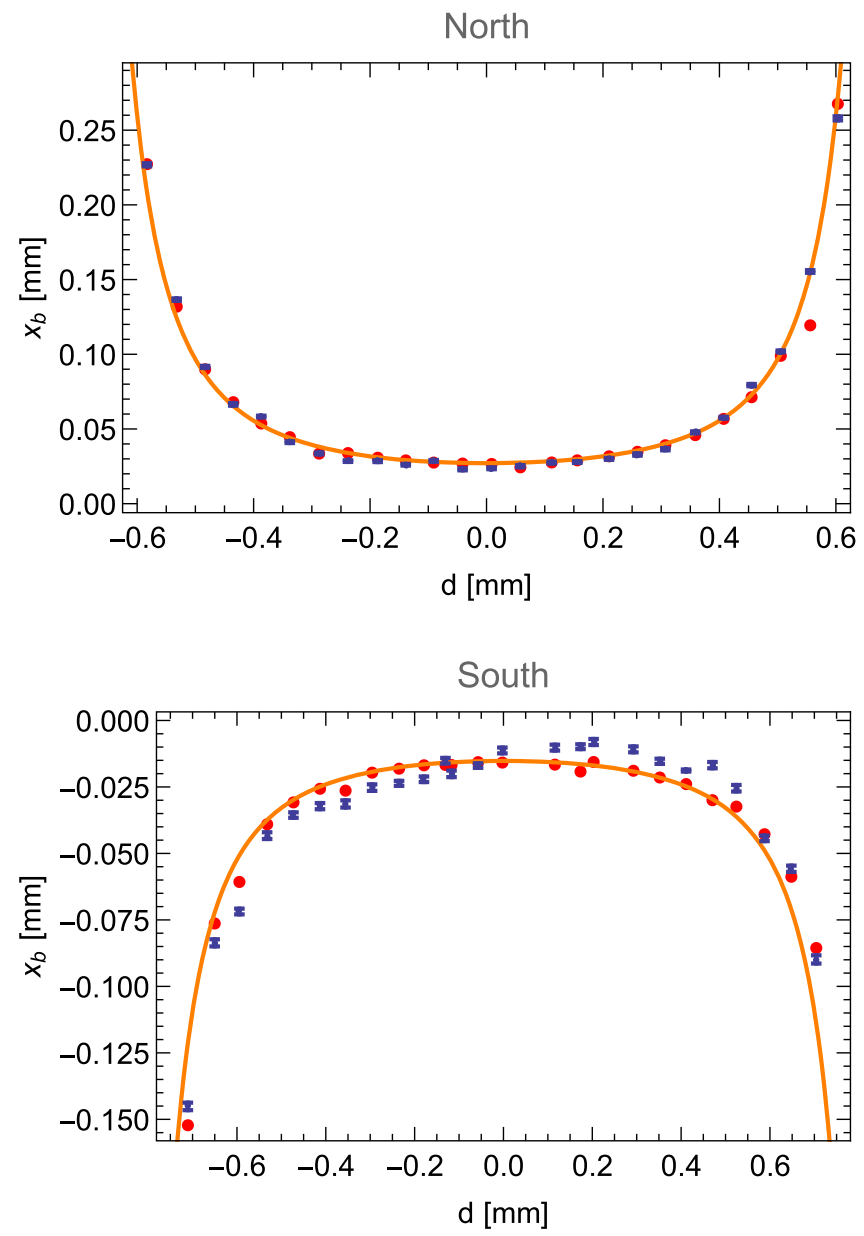

FIG. 7. Measurement of downstream beam offset, $x_{b}$, vs. jaw tilt parameter, $d$, for the North (top plot) and South (bottom plot) dechirper jaws. The blue plotting symbols give the measurement points, after they have been shifted vertically by $-x_{b 0}$, horizontally by $-d_{0}$, of the fitted parameters. The red curve gives the theoretical result assuming $b$ is fixed at $b=\langle b\rangle=0.70$ (0.86) $\mathrm{mm}$ for the North (South) case. The red symbols give the more accurate theoretical result, obtained from the twoparameter fit to the measured $\left(\delta b_{i}, d_{i}\right)$.

$d=0.55 \mathrm{~mm}$-is one for which $\delta b$ (and thus $b$ ) turned out to be different. The fitting parameters were almost the same for the two fitting methods, with (in the two variable case) $\langle b\rangle=b_{0}+\langle\delta b\rangle=0.70 \mathrm{~mm}$. A visual measure of the agreement between data and theory is the distance between corresponding blue and red symbols. We see that the fit (red symbols) to the data (blue symbols) is good, and that both agree well with the theory assuming constant $b$ (red curve).

The corresponding South results are given in the bottom plot of Fig. 7, and the fitting parameters can be found in Table II. The estimated error in measured $x_{b}$ is $\sim 1.5 \mu \mathrm{m}$, and we see that the error bars have started to become visible. Here the average beam offset for all data is $\langle b\rangle=0.86 \mathrm{~mm}$. We find here more variation in $\delta b_{i}$ obtained from the LVDT readings (see Fig. 10 in 
TABLE II. For the four cases, the fitted: shift in average beam offset, $b_{0}$; average beam offset from jaw, $\langle b\rangle$; shift in downstream (BPM 590) measurement $x_{b 0}$ or $y_{b 0}$; shift in tilt parameter, $d_{0}$; standard deviation of residuals of fit, $\sigma_{r}$.

\begin{tabular}{lrcccc}
\hline \hline Case & $b_{0}[\mathrm{~mm}]$ & $\langle b\rangle[\mathrm{mm}]$ & $x_{b 0}\left(y_{b 0}\right)[\mu \mathrm{m}]$ & $d_{0}[\mathrm{~mm}]$ & $\sigma_{r}[\mu \mathrm{m}]$ \\
\hline North & 0.14 & 0.70 & -8 & -0.42 & 8 \\
South & -0.06 & 0.86 & -23 & 0.15 & 7 \\
Top & 0.22 & 1.14 & 13 & 0.05 & 1 \\
Bottom & 0.07 & 1.26 & 1 & 0.04 & 1 \\
\hline \hline
\end{tabular}

Appendix B, the red symbols). In the wake kick plot (the bottom of Fig. 7) we see that theory and data agree quite well, though not so well as before. In addition, in the data (the blue symbols) we notice a significant asymmetric
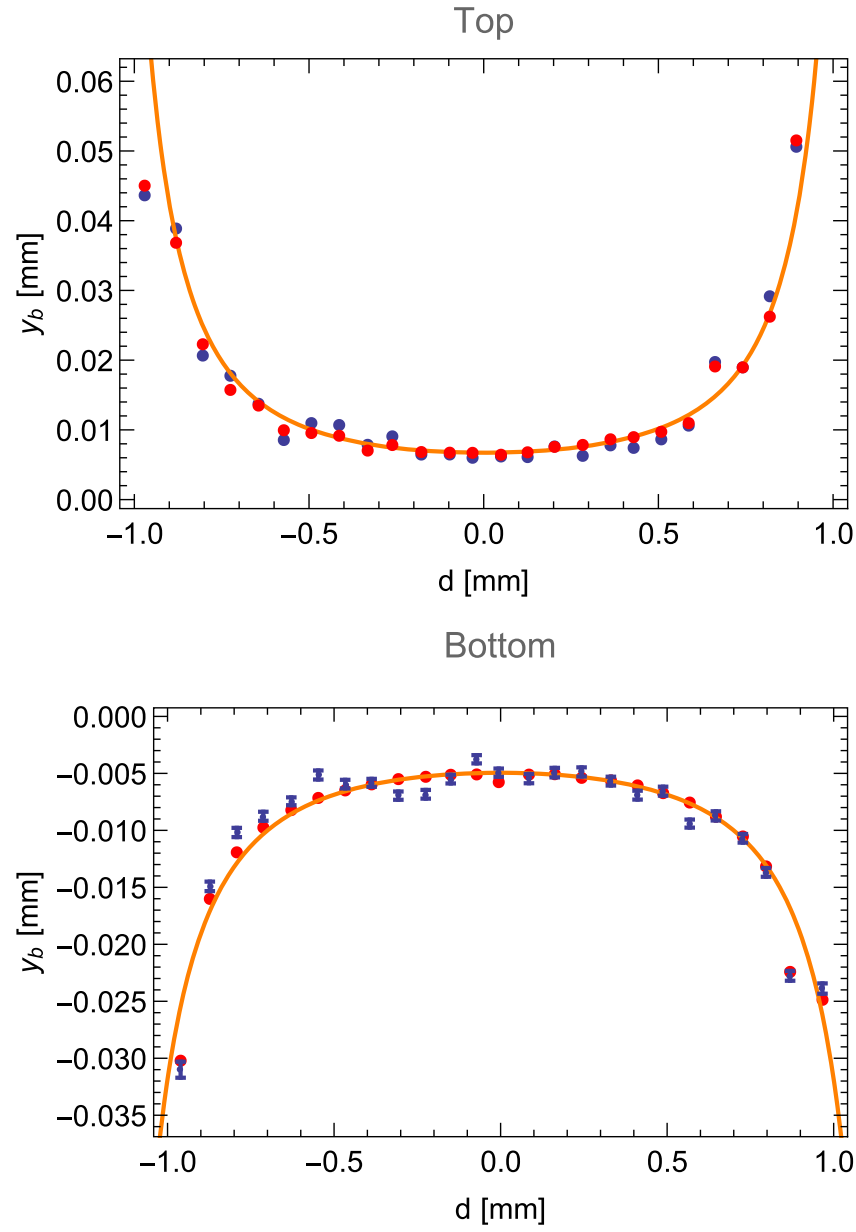

FIG. 8. Measurement of downstream beam offset, $y_{b}$, vs. jaw tilt parameter, $d$, for the Top (top plot) and Bottom (bottom plot) dechirper jaws. The blue plotting symbols give the measurement points, after they have been shifted vertically by $-y_{b 0}$, horizontally by $-d_{0}$, of the fitted parameters. The red curve gives the theoretical value assuming $b$ is fixed at $b=\langle b\rangle=1.14$ (1.26) $\mathrm{mm}$ for the Top (Bottom) case. The red symbols give the more accurate theoretical result, obtained from the two-parameter fit to the measured $\left(\delta b_{i}, d_{i}\right)$. component in $d$ dependence, one that seems to be nonphysical and is not understood. One can note, however, that the red plotting symbols are all on, or very near to, the red curve, suggesting that misorientation of the plate is not the cause of the discrepancy.

The Top and Bottom results are shown in Fig. 8 (with the corresponding $\delta b_{i}$ vs $d_{i}$ from the readbacks given in Fig. 10; see the gold and green symbols). The estimated rms error in measured $y_{b}$, for both cases, is $\sim 5 \mu \mathrm{m}$. The fitting parameters can again be found in Table II; here $\langle b\rangle=1.14$ (1.26) $\mathrm{mm}$ for the Top (Bottom) measurement. We see that the agreement with theory in both cases is good. Note that there are several red-blue pairs of points that agree well to each other though they are off the red curve; this indicates that $\delta b$ has shifted, while nevertheless, theory and measurement are in good agreement.

Table II summarizes the fitted results for all four plate measurements. The last column in the table gives the standard deviation of the residuals of the fit to the data, which we see are small in all cases, $10 \mu \mathrm{m}$ or less. Finally, note that the fitted shifts in downstream BPM reading, $x_{b 0}$ and $y_{b 0}$, give the expected null readings; i.e., the readings when the dechirper plate is moved far away from the beam. From the spread of the results in the table, we estimate that the accuracy in this parameter is $\pm 10 \mu \mathrm{m}$.

\section{CONCLUSIONS}

The dechirper jaws of the RadiaBeam/SLAC dechirper at the Linac Coherent Light Source (LCLS) are independently adjustable at both ends, and it is difficult to avoid leaving residual (longitudinal) tilt in them during dechirper set-up and alignment. In this report we present a modelindependent method of removing unknown tilt in a dechirper jaw, and demonstrate by experiment that it works well.

In addition, we derive an explicit analytical formula for the transverse wake kick of a single dechirper plate, as function of plate offset and tilt angle with respect to the beam orbit. We present wake measurements with the different LCLS dechirper jaws and show that, for the kick factors, agreement with theory is excellent. Compared to previously reported single plate wake measurements that assumed the tilt angle was small and not important [4], the measurements reported here are a more sensitive test and stronger confirmation of the theory.

Having demonstrated the accuracy and sensitivity of this measurement procedure for orienting a dechirper jaw, we propose incorporating it routinely in the set-up and alignment of the dechirper at the LCLS. The procedure is relatively simple and quick to perform.

In Appendix A we derive an explicit analytical formula for the longitudinal wake kick of a single dechirper plate, as function of plate offset and tilt angle with respect to the beam orbit. 


\section{ACKNOWLEDGMENTS}

We thank the SLAC operators for helping in the experiments. Work supported in part by the U.S. Department of Energy, Office of Science, Office of Basic Energy Sciences, under Contracts No. DE-AC02-76SF00515 and No. DESC0009550.

\section{APPENDIX A: LONGITUDINAL EFFECT}

For a uniform bunch distribution, the loss factor-the average of the longitudinal bunch wake-is given by [4]

$$
\varkappa=\left(\frac{Z_{0} c}{4 \pi b^{2}}\right) f_{z}\left(\frac{\ell}{s_{0 l}}\right)
$$

with

$$
s_{0 s}=\frac{2 b^{2} t}{\pi \alpha^{2} p^{2}}
$$

and

$$
f_{z}(\zeta)=\frac{2}{\zeta}\left(1-\frac{6}{\zeta}\right)+e^{-\sqrt{\zeta}}\left[\frac{4}{\zeta}\left(1+\frac{3}{\zeta}\right)+\frac{12}{\zeta^{3 / 2}}\right]
$$

For a plate with a small angle tilt, with average offset $b$ and offset at the ends $b \pm d$, we approximate the total loss factor as

$$
\varkappa_{t}=\frac{1}{2 d} \int_{b-d}^{b+d} \varkappa\left(b^{\prime}\right) d b^{\prime} .
$$

Substituting in Eq. (A1), and performing the integral we obtain

$$
\varkappa_{t}=\left(\frac{Z_{0} c}{8 \pi d}\right)\left[g_{z}(b+d)-g_{z}(b-d)\right]
$$

with

$$
g_{z}(x)=\frac{2 x}{\xi_{z}^{2}}\left[-2 x^{2}+2 x e^{-\xi_{z}^{1 / 2} / x}\left(x+\xi_{z}^{1 / 2}\right)+\xi_{z}\right],
$$

where

$$
\xi_{z}=\frac{\pi \alpha^{2} p^{2} \ell}{2 t}
$$

\section{APPENDIX B: MEASUREMENT DETAILS}

\section{Dechirper plate movers and readouts}

For the RadiaBeam/LCLS dechirper installed in the LCLS, Fig. 9 gives a sketch of the adjustment and

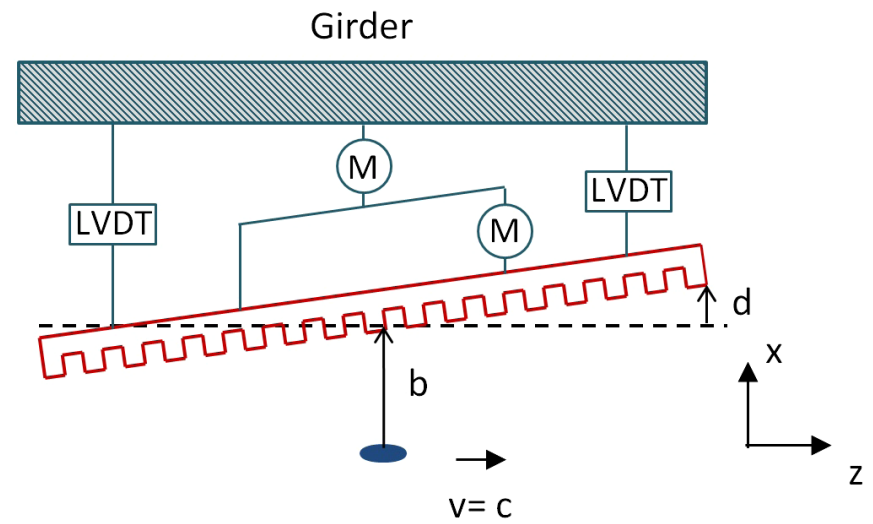

FIG. 9. Sketch of the position correction ( $\mathrm{M}$ stands for "mover") and read-back (LVDT sensors) for each dechirper plate. As suggested in the figure, one mover controls the average plate offset, and the other one, just the downstream end. The LVDTs measure offsets at the ends of the plate.

read-back system for each jaw. There are two movers ("M"), and two LVDT (linear variable differential transformer) position sensors, which are located near both ends of the jaw.

\section{LVDT readings during measurements}

For the measurements presented in the main text, we show the LVDT readings, transformed to offset parameter $\delta b_{i}$ and tilt parameter $d_{i}$, in Fig. 10.

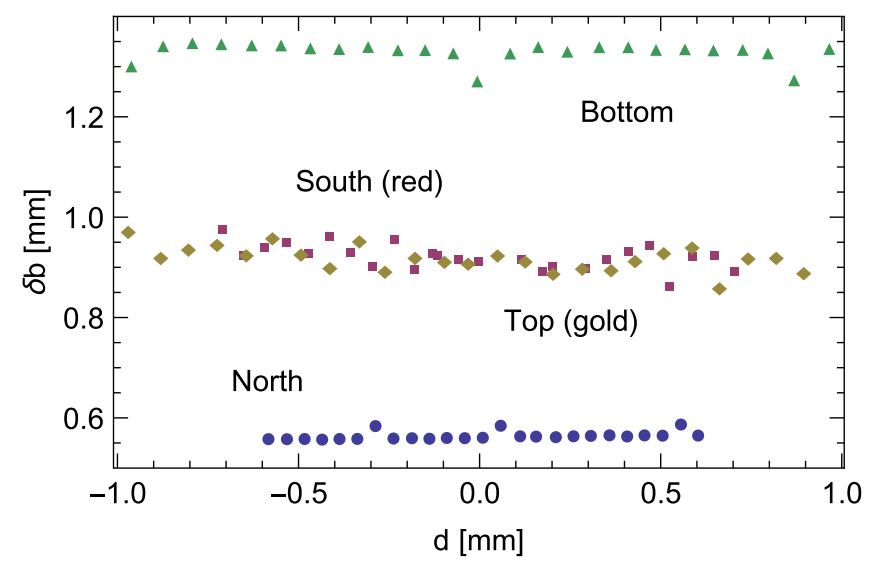

FIG. 10. The measured variation in average offset parameter, $\delta b_{i}$, corresponding to tilt parameter $d_{i}$, for all the measurements. The abscissas were shifted by the fitted $d_{0}$, to be consistent with the main measurement plots, Figs. 7 and 8 above. 
[1] P. Emma et al., First lasing and operation of an angstromwavelength free-electron laser, Nat. Photonics 4, 641 (2010).

[2] A. Lutman et al., Fresh-slice multi-colour x-ray freeelectron lasers, Nat. Photonics 10, 745 (2016).

[3] M. Guetg et al., Commissioning of the RadiaBeam/SLAC dechirper, in Proceedings of the 4th International Particle Accelerator Conference, IPAC2016 (Busan, Korea, 2016), p. 824.

[4] J. Zemella, K. Bane, A. Fisher, M. Guetg, Z. Huang, R. Iverson, P. Krejcik, A. Lutman, T. Maxwell, A. Novokhatski, G. Stupakov, Z. Zhang, M. Harrison, and M. Ruelas, Measurement of wake-induced electron beam deflection in a dechirper at the Linac Coherent Light Source, Phys. Rev. Accel. Beams 20, 104403 (2017).

[5] P. Emma, M. Venturini, K. L. F. Bane, G. Stupakov, H.-S. Kang, M. S. Chae, J. Hong, C.-K. Min, H. Yang, T. Ha, W. W. Lee, C. D. Park, S. J. Park, and I. S. Ko, Experimental Demonstration of Energy-Chirp Control in Relativisitc Electron Bunches Using a Corrugated Pipe, Phys. Rev. Lett. 112, 034801 (2014).

[6] H. Deng, M. Zhang, C. Feng, T. Zhang, X. Wang, T. Lan, L. Feng, W. Zhang, X. Liu, H. Yao, L. Shen, B. Li, J. Zhang, X. Li, W. Fang, D. Wang, M.-e. Couprie, G. Lin, B. Liu, Q. Gu, D. Wang, and Z. Zhao, Experimental
Demonstration of Longitudinal Beam Phase-Space Linearizer in a Free-Electron Laser Facility by Corrugated Structures, Phys. Rev. Lett. 113, 254802 (2014).

[7] F. Fu, R. Wang, P. Zhu, L. Zhao, T. Jiang, C. Lu, S. Liu, L. Shi, L. Yan, H. Deng, C. Feng, Q. Gu, D. Huang, B. Liu, D. Wang, X. Wang, M. Zhang, Z. Zhao, G. Stupakov, D. Xiang, and J. Zhang, Demonstration of NonlinearEnergy-Spread Compensation in Relativistic Electron Bunches with Corrugated Structures, Phys. Rev. Lett. 114, 114801 (2015).

[8] C. Lu, F. Fu, T. Jiang, S. Liu, L. Shi, R. Wang, L. Zhao, P. Zhu, Z. Zhang, and D. Xiang, Time-resolved measurement of quadrupole wakefields in corrugated structures, Phys. Rev. Accel. Beams 19, 020706 (2016).

[9] K. Bane, G. Stupakov, and I. Zagorodnov, Analytical formulas for short bunch wakes in a flat dechirper, Phys. Rev. Accel. Beams 19, 084401 (2016).

[10] K. Bane, G. Stupakov, and I. Zagorodnov, Wakefields of a beam near a single plate in a flat dechirper, Report No. SLAC-PUB-16881, 2016.

[11] Y. Ding, C. Behrens, P. Emma, J. Frisch, Z. Huang, H. Loos, P. Krejcik, and M-H. Wang, Femtosecond X-ray pulse temporal characterization in free-electron lasers using a transverse deflector, Phys. Rev. ST Accel. Beams 14, 120701 (2011). 